INPLASY

PROTOCOL

To cite: Yu et al. Association between intratumor heterogeneity and prognosis in solid tumor patients: a systematic review and metaanalysis. Inplasy protocol 202060107. doi: 10.37766/inplasy2020.6.0107

Received: 29 June 2020

Published: 29 June 2020

Corresponding author: Gang Liu

Ig1059@tmu.edu.cn

Author Affiliation:

Tianjin Medical University

General Hospital

Support: No.

Review Stage at time of this submission: Formal screening of search results against eligibility criteria.

Conflicts of interest: The authors have no financial or other conflicts of interest to disclose.

\section{Association between intratumor heterogeneity and prognosis in solid tumor patients: a systematic review and meta-analysis}

Yu, T1; Zheng, Z2; Gao, X33 Zhao, X4; Li, C5; Liu, G6.

Review question / Objective: How does intratumor heterogeneity impact on prognosis of solid tumor patients? P:solid tumor patients; I: none; C: high and low heterogeneity; $O$ : overall survival, progression free survival, disease free survival.

Condition being studied: In solid tumors, the heterogeneity of each patient's tumor is different, and the heterogeneity of the tumor is a manifestation of tumor evolution, which means that the biological behavior of the tumor may be different. Therefore, heterogeneity within the tumor may affect the prognosis to some extent.At present, we have not found a meta-analysis of related studies.

Information sources: We used intratumor heterogeneity and prognosis as keywords, and searched in four databases: PubMed, EMBASE, Cochrane Library, and Web of Science. The search deadline is February 15, 2020. The article language and country are not limited. The conference studies is also included.

INPLASY registration number: This protocol was registered with the International Platform of Registered Systematic Review and Meta-Analysis Protocols (INPLASY) on 29 June 2020 and was last updated on 29 June 2020 (registration number INPLASY202060107).

\section{INTRODUCTION}

Review question / Objective: How does intratumor heterogeneity impact on prognosis of solid tumor patients? P:solid tumor patients; I: none; C: high and low heterogeneity; 0 : overall survival, progression free survival, disease free survival.

Condition being studied: In solid tumors, the heterogeneity of each patient's tumor is different, and the heterogeneity of the tumor is a manifestation of tumor 
evolution, which means that the biological behavior of the tumor may be different. Therefore, heterogeneity within the tumor may affect the prognosis to some extent.At present, we have not found a meta-analysis of related studies.

\section{METHODS}

Search strategy: We used intratumor heterogeneity and prognosis as keywords, and searched in four databases: PubMed, EMBASE, Cochrane Library, and Web of Science. The search deadline is February 15,2020 . The article language and country are not limited. The conference studies is also included.

Participant or population: solid tumor patients.

Intervention: Not applicable.

Comparator: High intratumor heterogeneity and low intratumor heterogeneity.

Study designs to be included: We will include randomized controlled trials and cohort studies to assess the impact of intratumor heterogeneity on prognosis.

Eligibility criteria: 1. Articles are generated by retrieval words 2 . Survival charts or written Hazard ratios and $95 \%$ confidence zones can be extracted by various methods.

Information sources: We used intratumor heterogeneity and prognosis as keywords, and searched in four databases: PubMed, EMBASE, Cochrane Library, and Web of Science. The search deadline is February 15, 2020. The article language and country are not limited. The conference studies is also included.

Main outcome(s): Overall survival, progression free survival, disease free survival.

Additional outcome(s): Not applicable.

Quality assessment / Risk of bias analysis: We use a specific scale for bias analysis.
Strategy of data synthesis: The data we synthesized included Hazard Ratio and $95 \%$ confidence intervals. We use the original data when there is a clear HR and $95 \%$ confidence interval in the original text. If there is only survival curve, we use the specific method to obtain Hazard Ratio and $95 \%$ confidence intervals.

Subgroup analysis: When $i^{2}$ is $\geq 50 \%$, we consider using subgroup analysis to reduce heterogeneity. At the same time, ITH may be affected by a variety of factors, including cancer type, stage, evaluation method, treatment, and sampling method. Therefore, we use subgroup analysis to assess the effect of intratumor heterogeneity on prognosis.

Sensibility analysis: Sensitivity analysis is used when the heterogeneity evaluation $i^{2}$ is greater than $\mathbf{5 0 \%}$.

Language: No.

Country(ies) involved: China.

Keywords: Intratumor heterogeneity; solid tumor; prognosis.

Dissemination plans: We will submitted this paper to a leading journal in this field.

Contributions of each author:

Author 1 - Tao Yu - Author 1 drafted the article and processed the image.

Author 2 - Zicheng Zheng - The author drafted the literature, conducted data extraction and statistical analysis, completed the quality assessment and produced the image.

Author 3 - Xin Gao - The author drafted the literature, extracted the data, and completed the quality assessment.

Author 4 - Xinyu Zhao - The author helped screen the studies based on including and excluding criteria.

Author 5 - Chunqiang Li - The author helped screen the studies based on including and excluding criteria.

Author 6 - Gang Liu - The author is a corresponding author and put forward the idea of this article. 